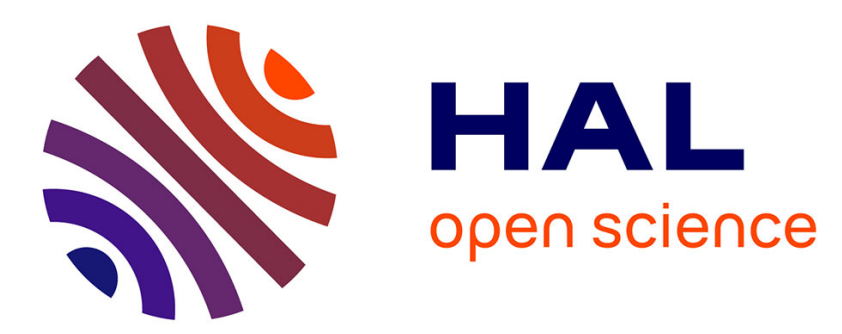

\title{
EER-LINC RF transmitter architecture for high PAPR signals using switched power amplifiers
}

Antoine Diet, Martine Villegas, Geneviève Baudoin

\section{To cite this version:}

Antoine Diet, Martine Villegas, Geneviève Baudoin. EER-LINC RF transmitter architecture for high PAPR signals using switched power amplifiers. Physical Communication, 2008, 1 (4), pp.248-254. 10.1016/j.phycom.2008.11.001 . hal-01691549

\section{HAL Id: hal-01691549 https://hal.science/hal-01691549}

Submitted on 25 Jan 2018

HAL is a multi-disciplinary open access archive for the deposit and dissemination of scientific research documents, whether they are published or not. The documents may come from teaching and research institutions in France or abroad, or from public or private research centers.
L'archive ouverte pluridisciplinaire HAL, est destinée au dépôt et à la diffusion de documents scientifiques de niveau recherche, publiés ou non, émanant des établissements d'enseignement et de recherche français ou étrangers, des laboratoires publics ou privés. 


\title{
EER-LINC high efficiency RF transmitter architecture using switched Power Amplifiers for high PAPR signals
}

\author{
A. Diet, M. Villegas, G. Baudoin
}

\begin{abstract}
Digital radio-communications uses nowadays different modulation schemes with high data rate possibilities. Designing a multi-standards linearised transmitter is an interesting goal for which OFDM (Orthogonal Frequency Division Multiplex) modulation is the worst case at the moment. Transmission of multi-carriers signals like OFDM ones are subject to high dynamic variation, quantified by the PAPR (Peak to Average Power Ratio) signal property. Non linearities occurring at the power amplification part implies the transmitter to be linearised for preserving the information integrity. This paper presents a new multi-standards linearised RF transmitter architecture based on both EER and LINC techniques. The goal is to cancel the influence of the high PAPR of an OFDM signal as soon as possible in the different steps of the transmitter. In order to keep the overall efficiency, the use of switched PAs is promoted, and being possible thanks to the constant (envelope) power property occurring by the EER-LINC method proposed. New circuits design are necessary and comparison with classical EER and LINC solutions are discussed.
\end{abstract}

Index Terms-Switched PA, EER, LINC, RF architecture, OFDM, linearization.

\section{INTRODUCTION}

$\mathrm{T}$ HIS paper focus is about digital wireless radiocommunications applications such as $3 \mathrm{G}+$ multimedia or WLAN data transfer. Nowadays, these applications need a high data rate which can be reached with multi-carriers modulation schemes. Orthogonal Frequency Division Multiplex (OFDM) is one solution widely used at the moment for example in IEEE 802.11a.

$$
C(t)=\Re\left\{\sum_{n=-N / 2}^{n=N / 2}\left[I_{n}(t)+j Q_{n}(t)\right] e^{j \omega_{0} t} e^{j n \Delta_{\omega} t}\right\}=\Re\left\{[I(t)+j Q(t)] e^{j \omega_{0} t}\right\}
$$

This type of signal is a sum of Quadrate Amplitude

Manuscript received 18 of July, 2007

A. Diet was with ESYCOM during this work and is now with L2S-DRE (UMR 8506 Univ. Paris-Sud 11, CNRS, Supélec) Supélec, plateau du Moulon, 3 rue Joliot Curie F-91192 Gif sur Yvette, France. antoine.diet@1ss.supelec.fr.

M. Villegas and G. Baudoin are with ESYCOM (EA 2552 groupe ESIEE, Univ. de Marne la Vallée, CNAM) groupe ESIEE, cité Descartes BP 99, 2 boulevard Blaise Pascal 93162 Noisy-le-Grand, France. g.baudoin@esiee.fr, m.villegas@esiee.fr.
Modulated (QAM) signals [6] and has a high dynamic variation of the radio-frequency $(\mathrm{RF})$ emitted signal $\mathrm{C}(\mathrm{t})$, this is characterized by the Peak to Average Power Ratio (PAPR) estimated in function of the number $\mathrm{N}$ of sub-carriers : PAPR $=10 . \log _{10}(N) . C(t)$ is expressed by equation (1) where $I_{n}$ and $\mathrm{Q}_{\mathrm{n}}$ denotes each sub-carriers $\mathrm{QAM}$ signals. Each subcarrier is spaced in frequency from $\omega_{0}$ by a shift of $\mathbf{n} \Delta_{\omega}$ as represented in figure 1 .

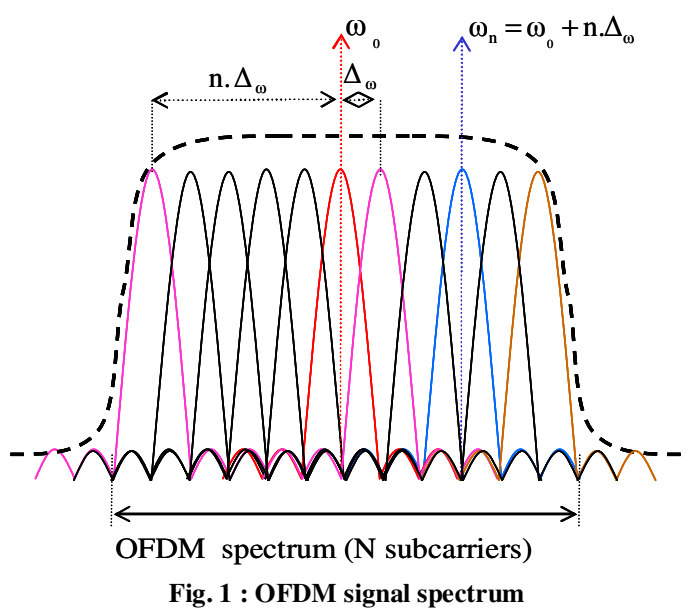

In wireless Radio-Frequency (RF) transmitter, the signal has to be power amplified before the emission. The higher is the PAPR, the more difficult is to keep the linearity of power amplification [3] [7] [10] [11] There are some possibilities to linearise the emitter but some of them lower the efficiency of the emitter [4] [5] [6] [8]. Some techniques based on the clipping and coding (e.g. PWM) of the envelope information can reduce the non linearities but distort the amplitude information at the same time [6] [8] [9]. Envelope Elimination and Restoration (EER) [1] and LInearisation with Non-linear Components (LINC) [2] are proposed methods that linearise the architecture and keep a high efficiency at the same time for high PAPR signals[7] [9] . These methods are supposed to be used with high efficiency switched Power Amplifiers (PAs). The use of such PAs combined inside linearization techniques is an interesting task and presents high advantages for future OFDM transmitters.

The idea is herein to combine advantages of switched PAs 
(high efficiency) with linearization techniques, in order to transmit high dynamic RF signals such as OFDM one. The idea is also to try to cancel the influence of the PAPR inside the transmitter architecture, avoiding at the same time any clipping or coding of the amplitude information. We want also to expose that the complexity of circuits added in the architecture is a small penalty compared to the potential performance of high efficiency linearization for these signals.

\section{LINEARIZATION TECHNIQUES AND SWITCHED PAS IN RF}

In this part, we will consider the emission of an RF OFDM signal. The OFDM modulation scheme is supposed to have enough sub-carriers, $\mathrm{N}$, to consider the PAPR high. This assumption will not enable the designer to take into account some linearization techniques based on correction such as feedback and feed-forward solutions because of the difficult tradeoffs between linearity and efficiency. We focus on high PAPR linearization techniques implying an important modification of the architecture: EER and LINC.
In a classical wireless transmitter, we can separate the RF architecture in three parts: the baseband signal generation, the RF transposition and the power amplification. Depending on the architecture, each part has outputs and inputs different depending on the signal decomposition. For example, in EER the signal is separated into amplitude and phase path and in LINC into two constant envelope signals thanks to a phase shift to be calculated. Fundamentally, the task of each part is conserved but is applied with different parameters on different signals paths. The figure 2 illustrates how the different parts are interconnected before we detail the signal processing inside each bloc. EER-LINC is based on EER and LINC techniques which we will summarize. We will underline tradeoffs of such techniques and then present the potential of using of high efficiency switched PAs. The EERLINC architecture will be detailed in the next part.

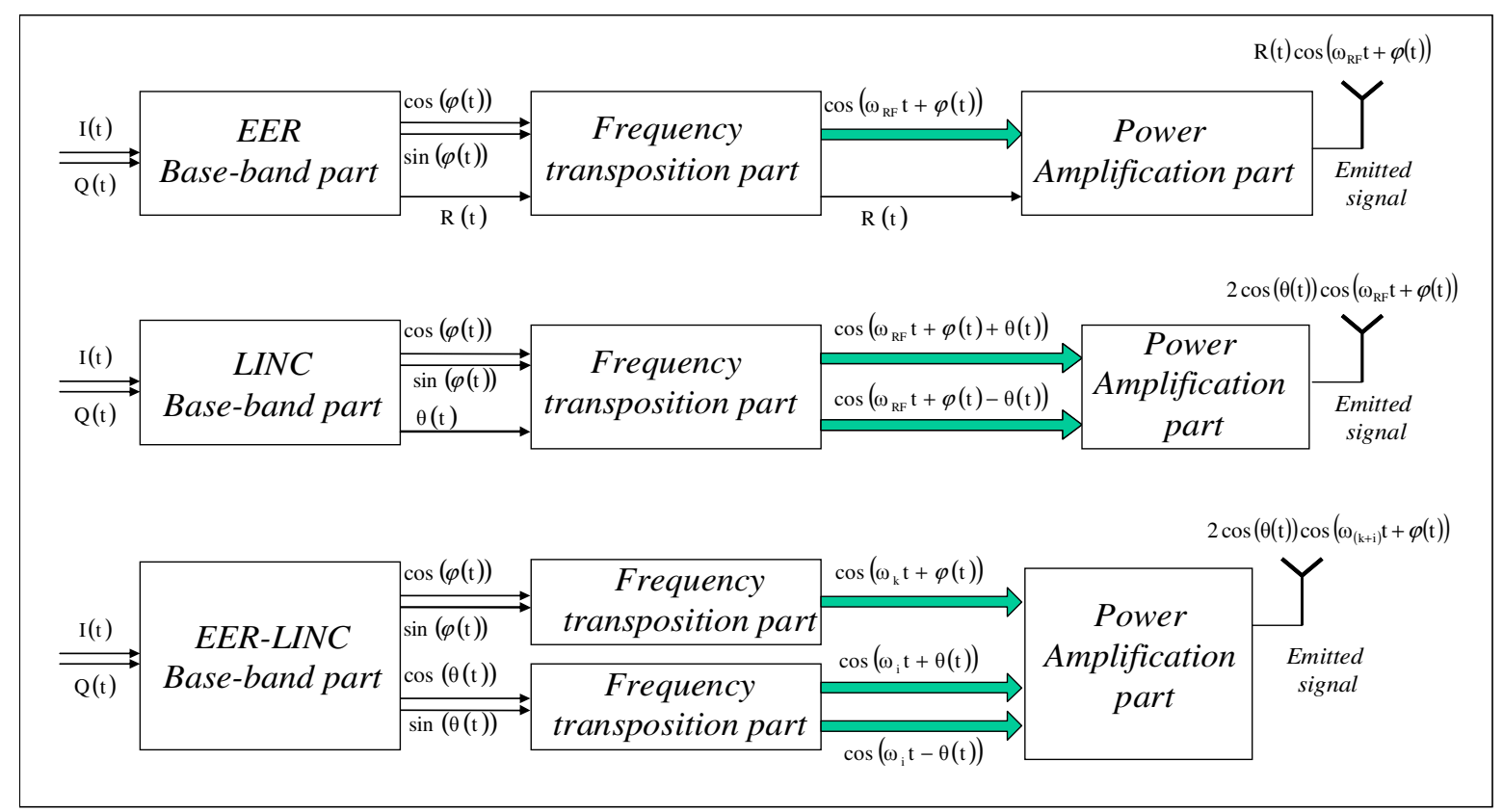

Fig. 2 : Decomposition of the RF transmitter architecture in three parts. Top is EER, middle is LINC and bottom is EER-LINC case.

\section{A. EER and LINC linearization techniques for RF emitter}

The EER technique [1] is based on a decomposition of the complex information $\mathrm{I}(\mathrm{t})+\mathrm{j} \cdot \mathrm{Q}(\mathrm{t})$ into its phase $\varphi(\mathrm{t})$ and magnitude $\mathrm{R}(\mathrm{t})$. Both of the information components are amplified: $\mathrm{R}(\mathrm{t})$ at the symbol frequency thanks to audio high efficiency techniques (example of the Pulse Width Modulation in the class $\mathrm{S}$ amplifier [6]) and $\varphi(\mathrm{t})$ at the RF carrier frequency (after transposition, see figure 2). The advantage is to use a high efficiency switched PA for amplification of the phase signal and to restore the amplitude information by modulation of the RF PAs supply (in a bloc schematic it is equivalent to a multiplication, but it will be detailed in next part). This results in an efficiency improvement of the transmitter depending on the power amplification of $\mathrm{R}(\mathrm{t})$ at a much lower frequency from the $\mathrm{RF}$ carrier. Possibilities of reaching high efficiency RF emission for OFDM signals were studied. The lack of EER is that the dynamic of the signal has still an influence on the architecture. The recombination of the amplitude and phase is subject to saturation and compression effects for high $R(t)$ PAPR value, see [9].

The LINC technique [2] is based on the decomposition of the complex information $\mathrm{I}(\mathrm{t})+\mathrm{j} . \mathrm{Q}(\mathrm{t})$ transposed at the $\mathrm{RF}$ carrier frequency into the sum of two constant envelope signals. This is accomplished via the calculus of a phase shift, $\theta(\mathrm{t})$ related to the amplitude information by $\cos (\boldsymbol{\theta}(\mathbf{t}))=\mathbf{R}(\mathbf{t})$, 
that has to be added and substracted to the phase information $\varphi(t)$. The recombination is done after high efficiency power amplification at RF frequency. The use of switched PAs is possible because of constant envelope property of the two signals. The lack of LINC is principally at the recombination step before the antenna. Reducing losses in the combiner is the point reducing directly the architecture efficiency [5] [11].

\section{B. Switched high efficiency RF PAs}

Both techniques, EER and LINC, use switched high efficiency PAs for different reasons. In the EER the switched PA is necessary because the emitted signal is proportional to the voltage supply, and enables supply modulation by the amplitude information $\mathrm{R}(\mathrm{t})$. In the LINC case, switched PAs are used to increase the efficiency and it is possible because the two signals have a constant envelope property. A switched PA is fundamentally based on the hard switching of a transistor where the dissipated power (DC) is minimized by improving the design of reactive elements $(\mathrm{L}, \mathrm{C})$ such as LC resonance, shunt capacitor, filters,... Its main particularity is the need of constant power at the input of the amplifier (no amplitude information). A consequence of hard switching is that the transistor saturation implies to filter the signal for transmitting the desired frequency component (RF carrier frequency) which amplitude is proportional to the voltage pulse, that is to say proportional to the supply voltage. Three switched PAs classes are particularly interesting for EER, LINC and EER-LINC architectures : class D, E and F. The design is discussed here in a MESFET based approach, which is totally coherent with other types of transistors (HBT,...).

Class D power amplifier (PA) is widely known in audio applications to be the impulse amplifier. The principle of its operation is to be driven by a square saturating signal and to produce square pulses at the output. This type of amplification is very useful for Pulse Width Modulation (PWM) technique [4][6]. Sinusoidal signal can be reproduced thanks to a resonant filter at the output.

The Class E PA was introduced by Sokal and carefully studied by Raab and Krauss [3][4][7]. Its principle is to reach the highest efficiency possible thanks to a parallel shunt capacitor and a series inductor as shown on figure 3 . Equations reported are those derived by the design of the class E PA in its optimal behaviour. The analysis was developed by Raab et al. and the influence of some imperfections due to non-ideal switching of the transistor can be found in [6] and [12]. Classes E PA enables the high efficiency possibility but is subject to a high variation of the voltage and current in the drain of the transistor (up to 3.56 $\mathrm{V}_{\mathrm{DC}}$ and $\mathbf{2 . 8 1} \mathrm{I}_{\mathrm{DC}}$ ). This implies an oversize design of the PA or the modification of reactive element conducting in a sub-optimal mode.

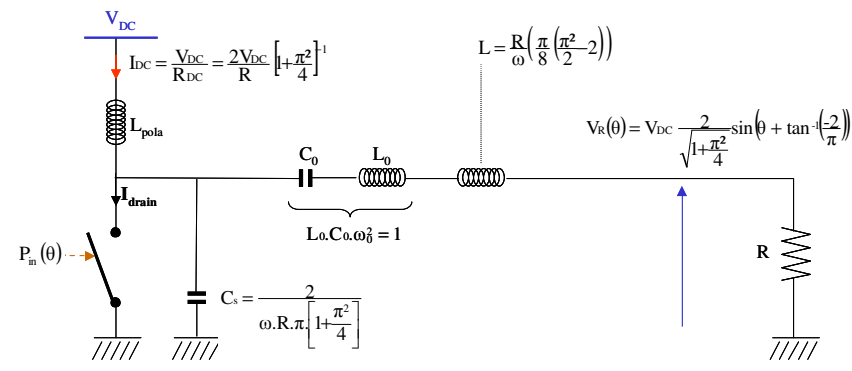

Fig. 3 : Class $E$ amplifier equations

Class F PA is another solution to improve the efficiency of the amplification. The principle is to modify the drain voltage and current with resonance of the signal harmonics in order to produce a 0 dissipated power (DC component of the power at the drain). Figure 4 illustrates the design of a class F PA with resonance of the $3^{\text {rd }}$ and $2^{\text {nd }}$ harmonic. Class $\mathrm{F}$ can reach a very important efficiency if the number of resonant harmonics is sufficient [7].

The choice between class $\mathrm{E}$ and class $\mathrm{F}$ is often due to the possibility/need of designing the LC output network. The factor of quality and the number of reactive elements are determinant in function of the frequency of operation. For these reasons, and taking into account the transistor switching imperfections, lowering the frequency of operation would help the designer to optimize the PA and reach the highest efficiency possible.

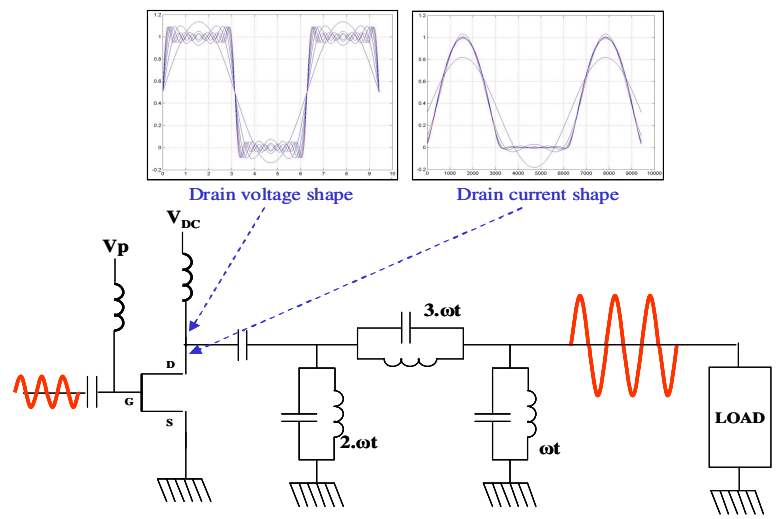

Fig. 4 : Class $\mathrm{F}$ principle for $2^{\text {nd }}$ and $3^{\text {rd }}$ harmonic resonance

\section{DESCRIPTION OF THE NEW COMBINED EER-LINC ARCHITECTURE USING SWITCHED PAS}

The new linearised architecture EER-LINC will now be detailed, following information given in figure 5. In the case of an OFDM signals, like $\mathrm{C}(\mathrm{t})$ defined by equation (1), the advantage of the structure is to cancel as soon as possible the influence of the PAPR on the PAs. This is accomplished by the EER and LINC cascaded decomposition that enables PAs to be driven at constant power input signals. Another benefit is the frequency of operation for all PAs which is lowered.

\section{A. Baseband Part}

The signal $\mathrm{C}(\mathrm{t})=\mathrm{I}(\mathrm{t})+\mathrm{jQ}(\mathrm{t})$, noted as $\mathrm{I}+\mathrm{jQ}$ for simplicity, is 
firstly decomposed in envelope, $\mathbf{R}=|\mathbf{I}+\mathbf{j} \mathbf{Q}|$, and phase, $\boldsymbol{\varphi}=\operatorname{Atan}(\mathbf{Q} / \mathbf{I})$, components such as in classical EER. The phase can be directly transmitted to the frequency transposition part in its complex form because it is by definition a constant envelope signal. Bandwidth property, as studied in [6] must be conserved. The envelope signal is the low frequency high dynamic source of non-linearity for the transmitter; its PAPR is directly proportional to the number of sub-carriers $\mathrm{N}$. The idea here is to decompose the signal $\mathrm{R}$ into a LINC structure. This is the same as using a LINC transmitter for a pure AM (or ASK) modulated signal. The result is the calculus of the parameter $\boldsymbol{\theta}$, as defined in figure 5 , which contains the information of R. Transmitting $\theta$ in its complex form enables the generation of constant envelope signals at the frequency transposition part. Simplification of the baseband processing, $\mathbf{R}=\cos (\boldsymbol{\theta})$, is possible if the condition on the amplitude of $\mathrm{I}$ and $\mathrm{Q}$ is respected $(\max <1)$. The baseband processing will produce four signals : $\cos (\varphi)$, $\sin (\varphi), \cos (\theta)$ and $\sin (\theta)$.

\section{B. Frequency transposition part}

The frequency transposition part for EER-LINC is to be split in two and the carrier's frequency choices are dependant of the power amplification part. First of all, the phase signal $\cos (\varphi)+\mathbf{j} \cdot \sin (\varphi)$ is transposed to a RF frequency $\omega_{k}$ by a classical IQ modulator. This is the phase path and remains unmodified compared to the EER, except for the value of $\omega_{\mathbf{k}}$.

To complete the LINC decomposition of the envelope R, the complex signal $\cos (\boldsymbol{\theta})+\mathbf{j} \cdot \sin (\boldsymbol{\theta})$ as to be transposed into two conjugate phase modulated signals at an intermediate frequency $\omega_{\mathbf{i}}$. The generation of such signals can be simply done by modifying a classical IQ modulator by inserting a buffer step and creating the two conjugate outputs as is illustrated on figure 5 . The sum of the two modulated signals will produce the envelope signal $\mathrm{R}(\mathrm{t})$ multiplied by the carrier signal at the frequency $\omega_{\mathrm{i}}$ as expressed by equation (2).

$$
\begin{aligned}
\cos \left(\omega_{\mathrm{i}} \mathrm{t}+\theta(\mathrm{t})\right)+\cos \left(\omega_{\mathrm{i}} \mathrm{t}-\theta(\mathrm{t})\right) & =2 \cdot \cos (\theta(\mathrm{t})) \cdot \cos \left(\omega_{\mathrm{i}} \mathrm{t}\right) \\
& =2 \cdot \mathrm{R}(\mathrm{t}) \cdot \cos \left(\omega_{\mathrm{i}} \mathrm{t}\right)
\end{aligned}
$$

The goal of the frequency transposition part is to produce only constant envelope signals. This is done at different frequency, one at $\omega_{\mathbf{k}}$ with the phase information and two "phase modulated conjugated" at $\omega_{\mathrm{i}}$ with the envelope information in a LINC decomposition. These three signals can now be amplified with high efficiency switched PAs and the major problem will be to recombine the information of $\mathrm{C}(\mathrm{t})$ at a desired carrier frequency $\boldsymbol{\omega}_{\mathbf{R F}}$.

For this part, only a classical IQ modulator and a modified one are to be used.

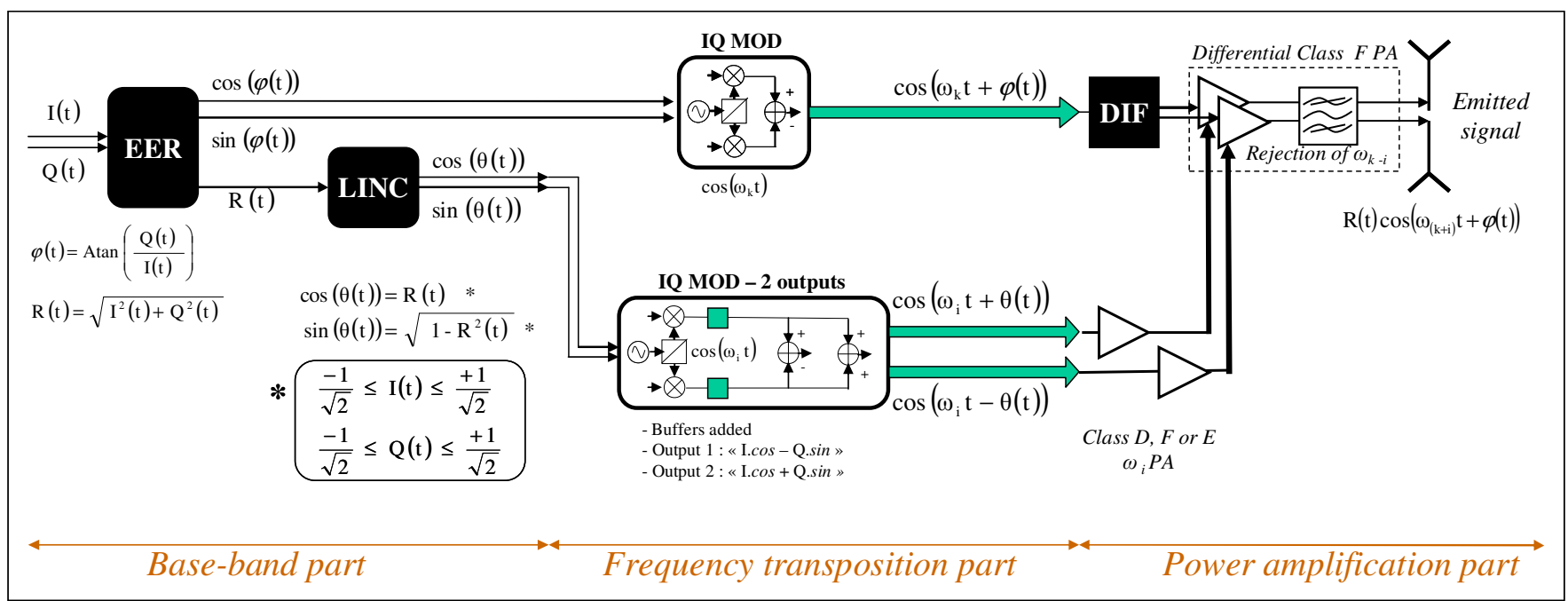

Fig. 5: Description of the EER-LINC architecture using high efficiency switched PAs

\section{Power amplification part}

The power amplification part is focused on the major problem of the architecture : the recombination of the phase and envelope information of $\mathrm{C}(\mathrm{t})$. The new idea is here to use the supply modulation as in the EER [6] but with a differential structure. As a consequence the output will be proportional to the sum of the two voltages supplies that are in fact the $\omega_{\mathbf{i}}$ amplified signals. This can be accomplished after the power amplification of such signals at the frequency $\omega_{i}$. As these signals are not RF, the class of operation can be
$\mathrm{D}, \mathrm{E}$ or $\mathrm{F}$ and the operation of amplification can easily reach high efficiency, as is supposed on figure 5 . The phase signal transposed to $\boldsymbol{\omega}_{\mathbf{k}}$ will thus need to be differential before the amplification. Using a switched differential pair with differentiation of the supply voltages will then enable the output $\mathrm{S}(\mathrm{t})$ to be the product of the input signal (the phase transposed at $\left.\omega_{\mathbf{k}}\right)$ by the sum of the $\omega_{\mathbf{i}}$ envelope signals. This results in equation (3), thanks to the results of equation (2), and without writing the amplification gain. As can be seen, the envelope information $\mathrm{R}$ is restored and the phase is transposed at both $\omega_{\mathrm{k}+\mathrm{i}}$ and $\boldsymbol{\omega}_{\mathrm{k}-\mathrm{i}}$ frequencies. It is also 
necessary to cancel one of the two signals without losses, in order to keep high efficiency power amplification. This can be done with LC filtering.

$\mathrm{S}(\mathrm{t})=\left[2 \cdot \mathrm{R}(\mathrm{t}) \cdot \cos \left(\omega_{\mathrm{i}} \mathrm{t}\right)\right] \cdot\left[\cos \left(\omega_{\mathrm{k}} \mathrm{t}+\varphi(\mathrm{t})\right)\right]$

$\mathrm{S}(\mathrm{t})=\mathrm{R}(\mathrm{t})\left[\cos \left(\left(\omega_{\mathrm{i}}+\omega_{\mathrm{k}}\right) \mathrm{t}+\varphi(\mathrm{t})\right)+\cos \left(\left(\omega_{\mathrm{k}}-\omega_{\mathrm{i}}\right) \mathrm{t}+\varphi(\mathrm{t})\right)\right]$
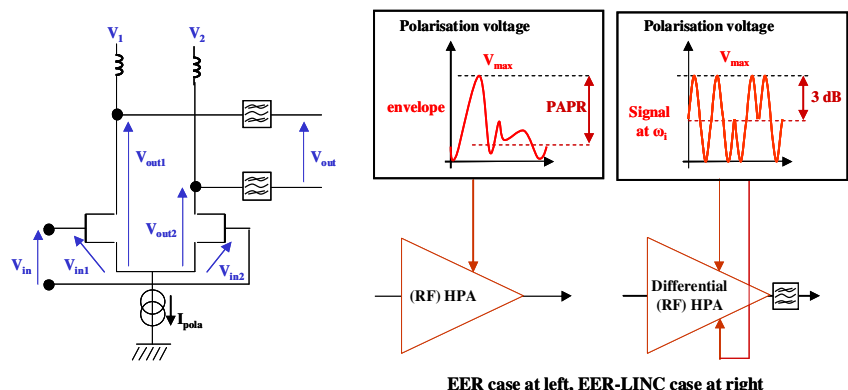

Fig. 6 : Switched differential pair with different supply voltages (left). Comparison between EER and EER-LINC supply modulation process.

The idea is so to transpose directly the information at $\boldsymbol{\omega}_{\mathbf{k}+\mathbf{i}}$ and amplifying it at the same time. The new structure used is based on a switched structure with LC filtering, as for the design of a class F PA, see figure 6. The goal is to design a differential switched pair that enables the transposition of its input signal at $\omega_{\mathrm{k}}$ to $\omega_{\mathrm{k}+\mathrm{i}}$. Simulations were done in [6] with a model of MESFET at a carrier frequency of $1 \mathrm{GHz}$ for $\omega_{\mathbf{k}}$ and a $50 \mathrm{MHz}$ frequency for $\omega_{\mathrm{i}}$. Figures 7 and 8 present the result of the co-simulation under HP-ADS. The capability to restore the amplitude information is shown on figure 7 and illustration of spectrum in the case of an OFDM signal is reported on figure $8\left(\mathrm{~d}_{1}\right.$ and $\mathrm{d}_{2}$ denotes the two signals at $\left.\omega_{\mathrm{i}}\right)$.
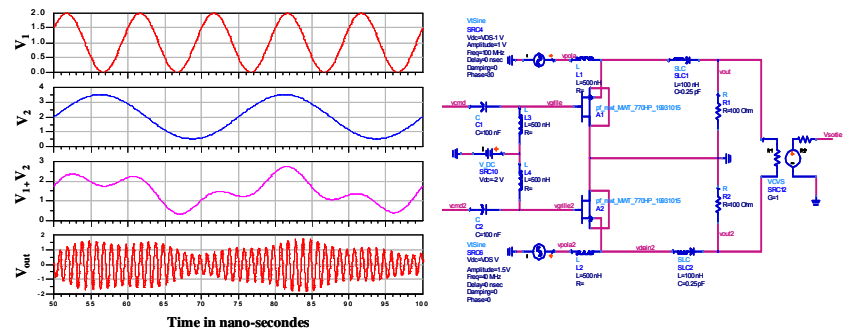

Fig. 7 : Simulation of the differential switched pair and illustration of the supply modulation on the two drains of the MESFET.

The possibility of using the new switched structure to provide amplification, transposition and restoration of amplitude information is simulated. This new structure is the key point of the EER-LINC architecture.
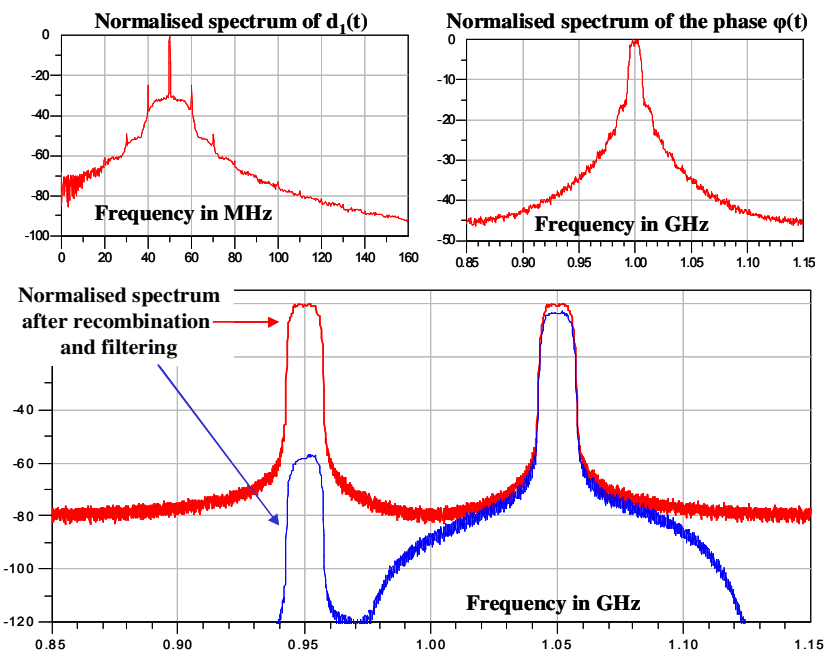

Fig. 8 : Spectrum of simulation with an OFDM signal and using the differential switched pair with LC filtering (co-simulation with ADS).

\section{ANALYSIS}

From an architectural point of view, the EER-LINC architecture implies different low frequency and high frequency circuits compared to EER and LINC.

At low frequency, EER-LINC needs the design of two high efficiency PAs for the envelope amplification but this is done with constant envelope signals and avoid the use of PWM or another envelope coding technique source of signal distortion [6]. Taking into account the dynamic is avoided and simplifies the overall design of the architecture. Designing of the modified IQ modulator by adding buffers seems not to be technically limiting and calculation of the LINC decomposition can be simplified using hypothesis of figure 5 .

At high efficiency, EER-LINC needs the design of the new PA structure called class $F$ differential switched pair that realises a transposition from $\omega_{\mathbf{k}}$ to $\omega_{\mathbf{k}+\mathbf{i}}$. This is not so penalising considering the difficulty for the design of the class E PA in the EER [9], which is limited by the PAPR, or the difficulty of the low losses combiner design in LINC. Another benefit is to potentially reduce the phenomenon of pulling between the antenna and the PA switching frequency, depending on the choice of $\boldsymbol{\omega}_{\mathbf{i}}$.

The futures works will be principally on two points. First is the design and measures at RF of the new structure "class $\mathrm{F}$ differential pair". The demonstration of the possibility to recombine envelope and phase information is the key point of the architecture. Second point is the generation of the four baseband signals needed in the case of an OFDM modulation scheme and the transposition at $\boldsymbol{\omega}_{\mathbf{k}}$ and $\boldsymbol{\omega}_{\mathbf{i}}$ of the phase and envelope information, implying the design of the modified IQ modulator.

\section{REFERENCES}

[1] L. R. Kahn. "Single Sideband Transmission by Envelope Elimination and Restoration". Proceedings of the I.R.E., 1952, pp. 803-806.

[2] D. C. Cox. "Linear amplification with non-linear components". IEEE transactions on Communications, Vol COM-23, pp 1942-1945, December 1974. 
[3] F. Raab et al. "Power Amplifiers and Transmitters for RF and Microwave". IEEE transactions on Microwave Theory and Techniques, Vol. 50, No. 3. Mars 2002, pp 814-826.

[4] H. Krauss. C. Bostian, F. Raab. "Solid State Radio Engineering", Wiley, New York, 1980.

[5] S. C. Cripps. "RF Powers Amplifiers for Wireless Communications". Artech House 1999

[6] A. Diet. "Etude des architectures EER pour les émetteurs de radio communications numériques $3 \mathrm{G}$ et au delà" $P h$. D. thesis report, Université de Marne la Vallée, France, 2005.

[7] F. Raab et al. "RF and Microwave Power Amplifier and Transmitter Technologies" High Frequency Electronics, May-November 2003, pp 22$36, p p$ 22-36, pp 34-48 and pp 38-49.

[8] W. Liu, J. Lau, R. Cheng. "Considerations on applying OFDM in Highly efficient PA". IEEE transactions on Circuits and Systems II, Vol. 46, No. 11, Nov. 1999, pp 1329-1335.

[9] A. Diet, C. Berland, M. Villegas, G. Baudoin. "EER architecture specifications for OFDM transmitter using a class E power amplifier". IEEE Microwave and Wireless Components Letters, pp 389-391, V-14 I-8, Août 2004.

[10] B. Razavi. "Challenges in Portable RF Transceiver Design". IEEE Circuits and Devices, pp 12-25, September 1996.

[11] B. Razavi. "RF Transmitter Architectures and Circuits". Proceedings of IEEE Custom Integrated Circuits Conference, pp 197-204, 1999.

[12] A. Hawaini, A. Diet, M. Villegas. "Amplification Classe E dans les architectures d'émetteurs linéarisées : influence des caractéristiques nonidéales". $15^{\text {èmes }}$ Journées Nationales Micro-Ondes, JNM 2007, Toulouse, France. 